\title{
THE HARP ELECTRON AND ION SENSOR ON THE PHOBOS MISSION
}

\author{
I.T. SZÜCS ${ }^{1)}$, I. SZEMEREY ${ }^{1)}$, P. KIRÁLY ${ }^{1)}$, W.E. SHARP ${ }^{2)}$, N.M. SHUTTE ${ }^{3)}$, T.E. CRAVENS ${ }^{2)}$, \\ T.I. GOMBOSI ${ }^{2)}$, K.I. GRINGAUZ ${ }^{3)}$, A.F. NAGY ${ }^{2)}$, S.M. SHERONOVA ${ }^{3)}$, S. SZENDRÖ ${ }^{\text {1), }}$ \\ M. TÁTRALLYAY ${ }^{1)}$, A. TÓ'TH ${ }^{1) \dagger}$ and M.I. VERIGIN ${ }^{3)}$ \\ 1) Central Research Institute for Physics, Budapest, Hungary \\ 2) Space Physics Research Laboratory, University of Michigan, Ann Arbor, USA \\ 3) Space Research Institute, Moscow, USSR
}

Received 25 May 1989 and in revised form 19 December 1989

The HARP instrument is a hyperbolic electrostatic analyzer working in the retarding potential mode. It is the lowest-energy member of the ESTER particle detector family. The energy range extends from $0.25 \mathrm{eV}$ to $850 \mathrm{eV}$ for both electrons and ions. The eight viewing sectors are arranged in a fan-shaped geometry in the antisolar hemisphere. They are simultaneously sampled while energy is stepped over a maximum number of 75 logarithmically spaced channels. The instrument is intended for thermal and suprathermal solar-wind electron observations during the cruise phase and - more importantly - for electron and ion observations in the Mars environment.

\section{Introduction}

Hyperbolic electrostatic analyzers [1] have been used for energy (or $E / Q$ ) filters in both laboratory and near-Earth space applications. In such analyzers the electrostatic field is of a simple quadrupole form, thus particle trajectories are easy to follow both analytically and by numerical simulation. The ratio of the deflecting potential to the energy of analyzed particles is large $(>1 \mathrm{~V} / \mathrm{eV})$ in comparison with the values of about 0.1 $\mathrm{V} / \mathrm{eV}$ typical for cylindrical, spherical and toroidal analyzers. Contact-potential problems which are often critical for low-energy particles are thus largely reduced, but of course the upper limit to the energy range is aiso lower than for conventional devices.

Previous space applications of hyperbolic analyzers were restricted to the terrestrial ionosphere. Suprathermal electrons play a fundamental role in planetary ionospheres. After creation by ionizing processes in the atmospheric gases, electrons lose their energy in collisions, and finally become a part of the thermal electron population. The measurement of the energy spectra at various altitujes with high accuracy provides an importanit set of data for comparison with theoretical models. For such purposes, several hyperbolic analyzers in the retarding potential mode, code-named HARP, have been launched on rockets into the ionosphere by a group at the Space Physics Research Laboratory (SPRL) of the University of Michigan, Ann Arbor, USA.

$\dagger$ Deceased in April 1989.
The role of suprathermal electrons and ions is also of considerable interest above the ionosphere, where direct collisions become less important, and particles arriving partly from the ionosphere and partly from the shocked solar wind become a part of a complex region of particles and fields. While in the terrestrial magnetosphere the planetary magnetic field is predominant, the situation around Mars is murn more balanced: thermal and suprathermal particle populations of planctary and solar wind origin as well as planetary and induced magnetic fields may play comparable roles.

Particle fluxes are expected to be anisotropic, therefore the original HARP device was upgraded into a sensor system having some directional sensitivity. Two sensor heads were arranged at right angle to each other, each of them distinguishing particles arriving from four angular sectors. The HARP devices flown on both Phobos spacecraft were built in the Central Research Institute for Physics, Budapest, Hungary, following a design developed from the above-mentioned rocketlaunched HARP versions of the SPRL. The MCP detectors were provided and tested by the Space Research Institute (IKKI), Moscow, USSR.

\section{The sensor system}

In the Phobos HARP sensor, trajectories of particles with different $E / Q$ values are separated electrostatically in a cavity enclosed by two hyperboloidal end-cap electrodes having the same potential (1 and 2 in fig. 1) and a ring-shaped hyperboloid ( 3 in fig. 1 , called the 


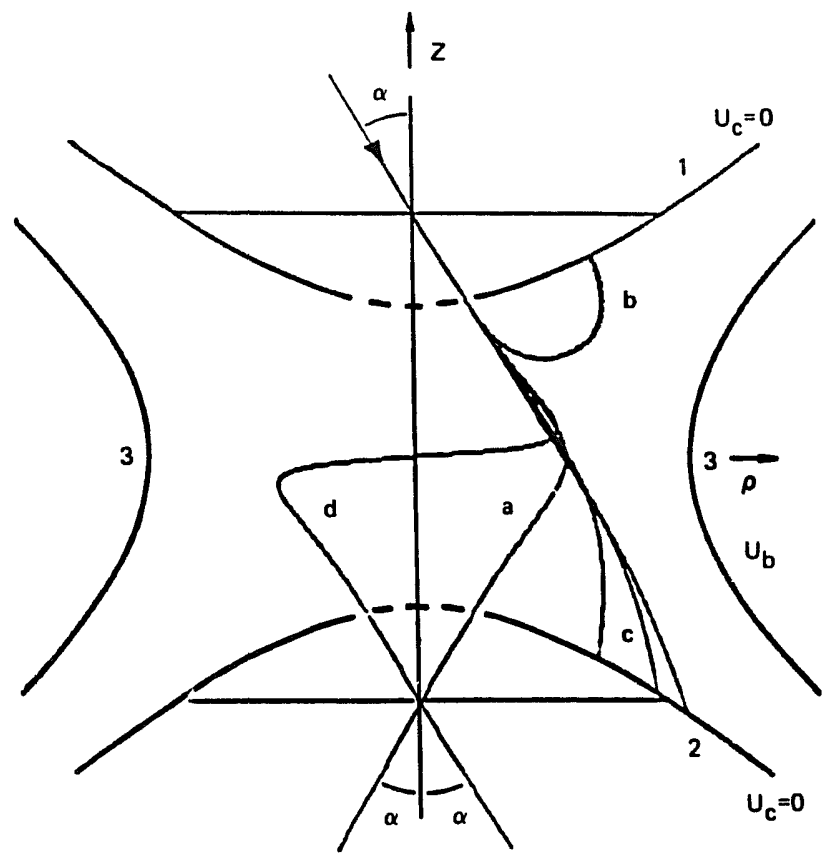

Fig. 1. Schematic of a hyperbolic analyzer with computersimulated particle trajectories.

body electrode) having a different potential. The energy spectrum is scanned by changing the potential of the body electrode in logarithmic steps following some predefined program. Particles that leave the cavity through the exit hole in four different directions are accelerated by a grid and fall on separate sections of a microchan- nel plate (MCP), and the resulting cascades reach separate anodes (fig. 2).

Inside the sensor cavity, an axially symmetric quadrupole field is produced with a saddle-point in the geometrical centre. Taking the cap potential $U_{c}=0$, the potential distribution inside the cavity can be expressed [1] as

$U(\rho, Z)=U_{b} \frac{\rho^{2}+2\left(Z_{c}^{2}-Z^{2}\right)}{\rho_{b}^{2}+2 Z_{c}^{2}}$,

where $\rho$ and $Z$ are cylindrical coordinates, $U_{\mathrm{b}}$ is the body potential, $\rho_{b}$ denotes the radius of the ring-shaped body electrode, and $Z_{c}$ is the half-distance between the two end-caps. From eq. (1) it is easy to check that the surface of the body electrode described by $\rho=\left(2 Z^{2}+\right.$ $\left.\rho_{b}^{2}\right)^{1 / 2}$ and those of the end-caps $Z= \pm\left(Z_{c}^{2}+\rho^{2} / 2\right)^{1 / 2}$ are really equipotential surfaces with potentials $U_{\mathrm{b}}$ and 0 , respectively. The value of the potential at the centre of the cavity is

$U(0,0)=U_{\mathrm{b}} \frac{2 Z_{\mathrm{c}}^{2}}{\rho_{\mathrm{b}}^{2}+2 Z_{\mathrm{c}}^{2}}$.

The central point $(0,0)$ is a saddle-point of the field. Around this point the potential can be described as

$U(\rho, Z)=U(0,0)+\frac{U_{\mathrm{b}}}{\rho_{\mathrm{b}}^{2}+2 Z_{\mathrm{c}}^{2}}\left(\rho^{2}-2 Z^{2}\right)$.

The spatial variation of the potential is thus described by a very simple quadratic function.

The equation of motion of particles in si:ch a potential field is separable in cylindrical coordinates. The

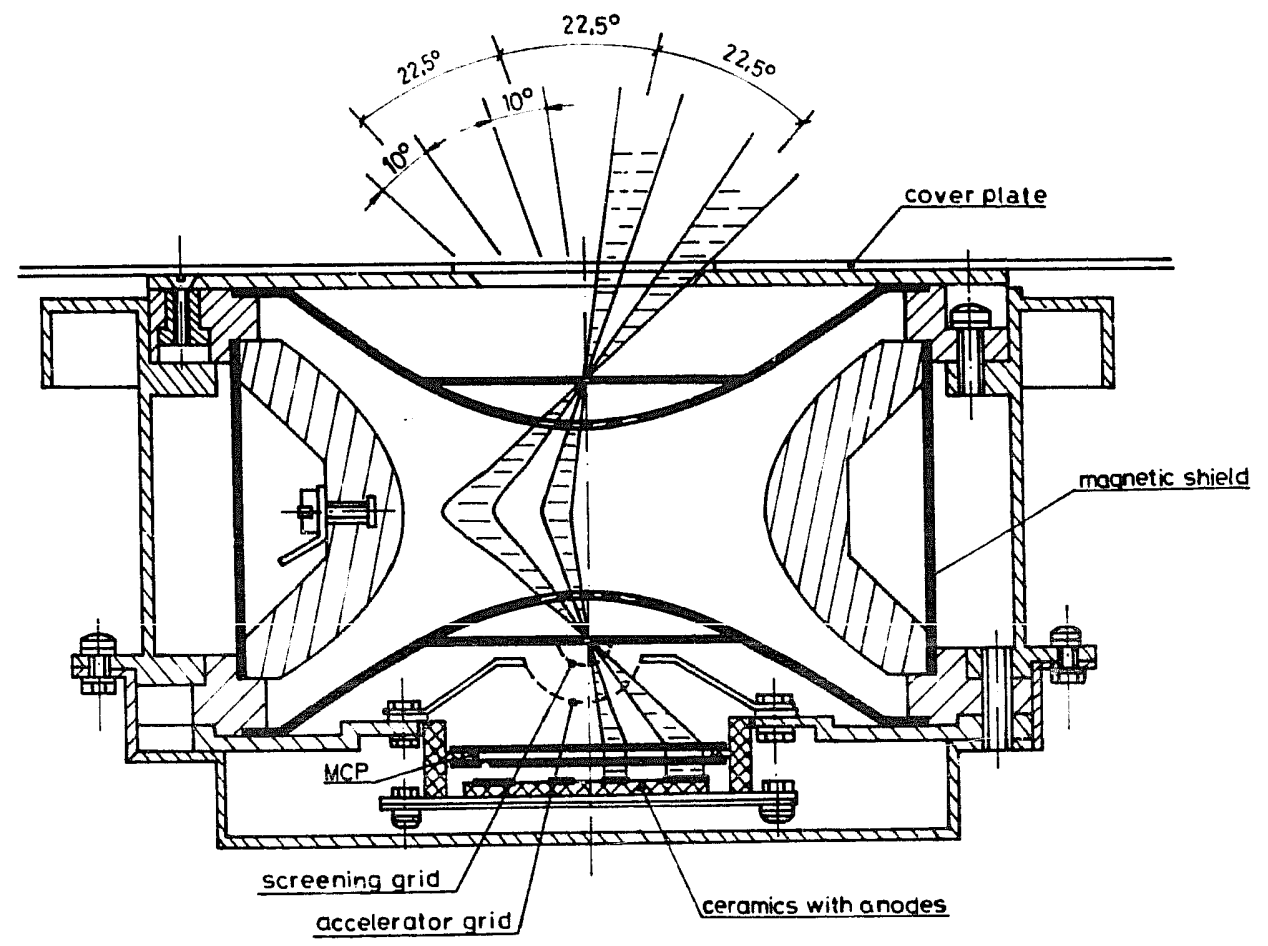

Fig. 2. Cross section of a sensor head of the Phobos HARP device. 
solutions are discussed in Shyn et al. [1] in some detail. Here we give only a short qualitative survey of the resulting trajectories.

In the configuration given in fig. 1 only particles of specific $E / Q$ values can get through from entry to exit hole. Curve $a$ shows such a trajectory. The particle is decelerated along the $Z$ axis as it approaches the $Z=0$ plane and is accelerated afterwards, while in the radial direction it always experiences a force pointing towards the $Z$ axis. A particle that does not have enough energy to penetrate the potential barrier at $Z=0$ is reflected (curve $b$ ). If the energy is too high, then the radiai force cannot sufficiently bend the trajectory towards the exit hole (curves $c$ ). For particles of opposite charge, the radial force points away from the axis. Although these particles always get through the $Z=0$ plane (in fact, are attracted towards it), they are deflected from the exit hole. Finally, there are so-called second-urder (and higher-order) trajectories (curve $d$ ) that get through the exit hole along somewhat more complicated curves than does the particle following the simple curve $a$ which has only one maximum in the radial coordinate. These second- and higher-order trajectories do contaminate the energy spectra and the directional distributions to some extent, but fortunately their contribution is usually negligible (not more than several percent). Firstorder trajectories - at least in the limit of infinitely small entry and exit holes - leave the sensor in a direction that corresponds to the mirror image of the entry direction.

It is an immediate consequence of the equations of motion that the trajectories inside the sensor cavity do not depend separately on the entry energy $E$, the charge $Q$ and the body potential $U_{\mathrm{b}}$, but only on the ratio $Q U_{\mathrm{h}} / E$. During calibration equivalent results are expected if the beam energy $E$ or the body potentiai $U_{\mathrm{b}}$ is varied (the latter possibility is technically more feasible). The sensor works in the retarding mode (i.e. particles are first decelerated, then accelerated) if $Q U_{\mathrm{b}}>0$. Thus both positive ion and negative electron spectra can be obtained by stepping $U_{\mathrm{b}}$ through positive and negative values, respeciively.

The entry direction of a trajectory can be described by two angles: the azimuth $\alpha$ in the plane of fig. 1, and the elevation $\delta$ in the perpendicular direction. P.ectangular slits in the end-cap electrodes define for $r$ viewing sectors (see fig. 2), each covering $10^{\circ}$ in $\alpha$ and $20^{\circ}$ in $\delta$. Because of symmetry, only two of the four are inequiv alent: the so-called inner and outer sectors (those closer to and farther from the axis). The acceptance properties of these two sectors are different.

Two sensor heads were arranged at right angle to each other. The fan-shaped joint solid angle coverage of the two sensors of the HARP instrument is shown in fig. 3. In the three-axis stabilized mode of the Phobos spacecraft the fan plane was perpendicular to the

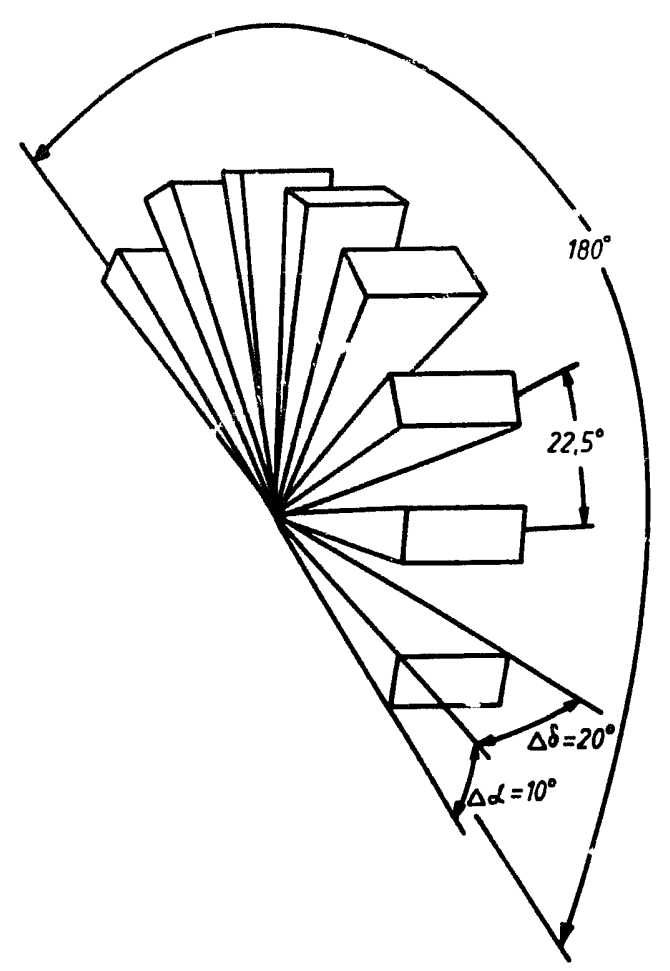

Fig. 3. Joint solid angle coverage of two sensor heads of a HARP device. In the three-axis stabilized mode of the Phobos spacecraft the fan plane was perpendicular to the ecliptic, with the central direction pointing anti-sunward.

ecliptic, with the central direction pointing anti-sunward.

Any external electric or magnetic field penetrating the sensor would distort the trajectories of very low energy particles. A good magnetic shielding is provided by a high-permeability shielding ring (CO-NETIC, $\mu>$ $50000)$ surrounding the body electrode. The end-caps are also made of the same material. The entry holes of the sensor system are surrounded by an iridium-coated Al plate of $30 \mathrm{~cm}$ diameter providing an equipotential neighbourhood. The exit hole of the sensor cavity is electrostatically shielded by a cylindrical screening grid, which also provides a virtually field-free drift space for the particles of different directions so that they can be spatially separated before being accelerated onto the MCP arrav (see fig. 2).

\section{Instrument characteristics and calibration}

The main characteristics of the HARP instrument developed for the two Phobos spacecraft are summarized in table 1 . The general properties listed in the first part need no explanation. The sensor characteristics are given in the second part. When appropriate. separate values are presented for the inner and outer sectors. 
Table 1

Instrument characteristics

\begin{tabular}{|c|c|c|}
\hline 1. Technical data & & \\
\hline Total mass & $3.5 \mathrm{~kg}$ & \\
\hline Volume & $4.61^{\circ}$ & \\
\hline Footprint & $12 \mathrm{~cm} \times 13.5 \mathrm{~cm}$ & \\
\hline Power & $4 \mathrm{~W}(+1 \mathrm{~W}$ for heater $)$ & \\
\hline Telemetry & & \\
\hline in low bit-rate mode & 240 bytes $/ 20 \mathrm{~min}$ & \\
\hline in high bit-rate mode & 480 bytes $/ \mathrm{min}$ & \\
\hline Temperature range & $-50^{\circ} \mathrm{C}$ to $+50^{\circ} \mathrm{C}$ & \\
\hline 2. Sensor characteristics & & \\
\hline Sensor type & hyperbolic electrostatic analyzer & \\
\hline Detected particles & electrons, ions & \\
\hline Number of sensors & 2 (with perpendicular axes) & \\
\hline Number of viewing sectors/sensor & 4 ( 2 inner, 2 outer $)$ & \\
\hline Number of energy steps & 75 (logarithmically spaced) & \\
\hline Time/step & $10 \mathrm{~ms}-1 \mathrm{~s}$ & \\
\hline Energy range & $0.25-850 \mathrm{eV}$ & \\
\hline Field of view/sector FW: & $20^{\circ} \times 10^{\circ}=6.99 \times 10^{-2} \mathrm{sr}$ & \\
\hline & irner sect $\mathrm{r}$ & outer sector \\
\hline$Q U_{h} / E$ conversion factor & $\overline{2.3}$ & 1.46 \\
\hline$\Delta E / E$ energy resolution $F W$ : & 0.1 & 0.28 \\
\hline FWHM: & 0.06 & 0.18 \\
\hline Projected area of entry hole & $2.76 \times 10^{-3} \mathrm{~cm}^{2}$ & $2.29 \times 10^{-3} \mathrm{~cm}^{2}$ \\
\hline Transmission factor & $8.8 \times 10^{-2}$ & $8.1 \times 10^{-3}$ \\
\hline MCP efficiency & 0.9 & 0.75 \\
\hline Effective energy-geometric factor & $1.3 \times 10^{-6} \mathrm{~cm}^{2} \mathrm{sr} \mathrm{eV} / \mathrm{eV}$ & $2.4 \times 10^{-7} \mathrm{~cm}^{2} \mathrm{sr} \mathrm{eV} / \mathrm{eV}$ \\
\hline Dynamic range & $1106.5 \times 10^{2}$ & /step \\
\hline
\end{tabular}

The relationship between incident particle energy and body potential is in first approximation characterized by a conversion factor and a $\Delta E / E$ energy resolution. For a given hody potential, the particles being in a specific phase space volume can enter the analyzer and get through to the MCP array to produce a signal on one of the anodes. The full width of the energy range ( $F W$ ) and the full width at half maximum (FWHM) characterize the energy resolution. The conversion factor gives the ratio of the body potential to the mean energy of particles detected in a viewing sector (assuming unit charge). The data in table 1 were based on numerical simulation.

The count rate $C$ measured by the instrument at the energy step corresponding to $E$ is related to the difforential intensity $j$ through the effective energy-geometric factor $G$ as $C=G E j$, where $G$ depends on the sensor geometry and is also proportional to the MCP efficiency. The factor $G$ for the inner and outer sectors was calculated in two main steps. First, a fuil tiansmission of the instrument was assumed over the full projected area of the entry hole, the full field of view and the FW/ energy resolution. Then by numerical simulation trajectories were calculated, starting from a number of points of the entry hole. from each point in a number of directions covering the field of view, and at a number of energics covering the full $\Delta E / E$ interval. The fraction of such trajectories that really can get through to the appropriate section of the MCP gives the transmission factor by which the first, crude estimate has to be corrected.

The energy-angle acceptance for both viewing secto: - was determined by trajectory tracing as explained above. Here the results of a simplified model calculation are presented for the inner sector. Particles were started from 10 different positions of the entry hole. Since 21 different azimuth angles were taken in the $\delta=0$ plane at $\Delta \alpha=0.5^{\circ}$ intervals, and $21 U_{h} / E$ values were applied for each angle at $0.5 \%$ intervals, the energy-angle matrix had $21 \times 21$ elements. At the entry hole each element's value was 10 . corresponding to the 10 starting positions. Iig. 4 shows the response matrix at exit. before the particles get to the appropriate section of the MCP. Line plots to the left and below represent summations over the matrix elements in angle and in energy, respectively. In this model about $17 \%$ of the original particles reach the detector and are counted $\checkmark$ th a probability given by the MCP efficiency. In a 

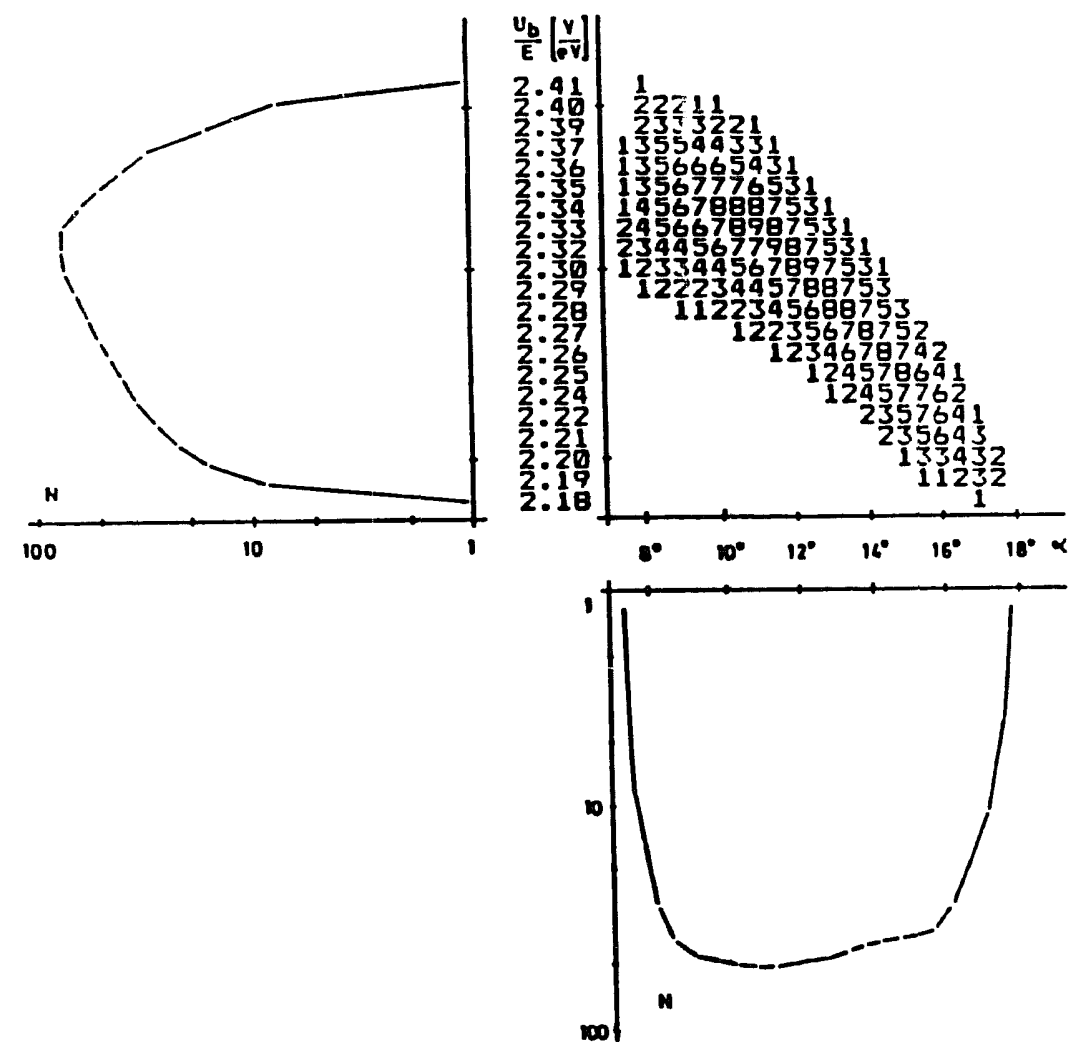

Fig. 4. Numerical simulation of the sensor response for the inner sector. The energy-angle acceptance is represented by a $21 \times 21$ matrix. The possible maximum value of an element in the response matrix is 10 . The curves present summations over the matrix elem.nts. Left: summation in angle, below: summation in energy.

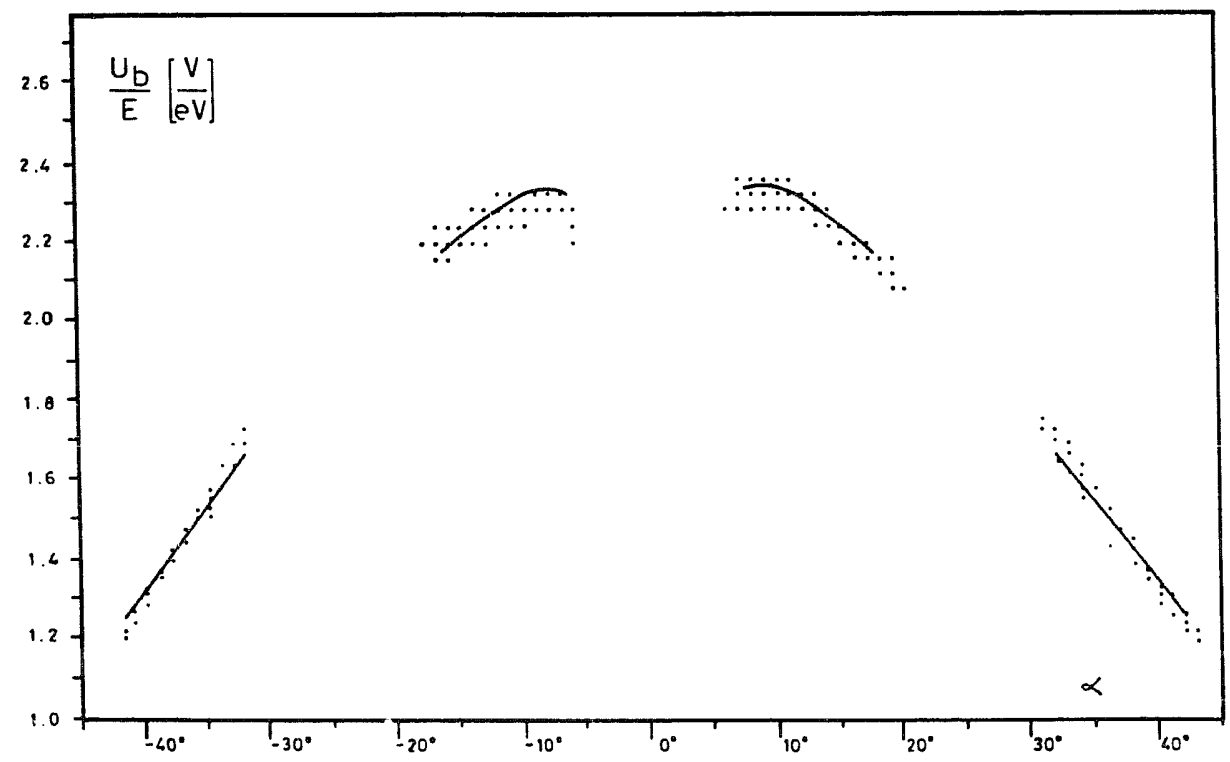

Fig. 5. Comparison of measured characteristics of HARP sensors to computer simulation. The sensors were exposed to $E=100 \mathrm{eV}$ electrons. For each value of the azimuth angle $\alpha$, the body potential $U_{\mathrm{b}}$ was swept until the maximum response was found. The $\alpha$, $U_{\mathrm{b}}, E$ pairs thus obtained are represented by dots, the simulated results by continuous lines. 
more realistic simulation, starting positions should be more uniformly distributed over the entrance hole (here they were located along one diameter of the hole), and the elevation angle $\delta$ should also be varied. This more detailed calculation would result in a further reduction of about a factor $\mathbf{2}$ in the transmission factor.

The small values of the transmission factors are partly due to the strong correlation between $\alpha$ and $U_{\mathrm{b}} / E$, and partly to the poor focusing properties. Both effects are even more important for the outer sectors.

The Phobos HARP sensors were calibrated for electrons at SPRL and for positive ions at The Marshall Space Flight Center, NASA, Huntsville, Alabama. Calibration results were generally consistent with numerical simulations. Unfortunately, technical conditions prevented a full calibration at all energies and in all positions of the sensors relative to the particle beam.

Fig. 5 illustrates one aspect of the comparison between simulated and measured data. Detector response to a $100 \mathrm{eV}$ electron beam was determined as a function of the azimuth angle $\alpha$. Four sensors were measured: two for Phobos 1 and two for Phobos 2. At each value of $\alpha$, the body potential $U_{b}$ was varied until the maximum response was found. Due to slight differences in the internal geometry of sensors, as well as to unavoidable deviations in the beam profile and in the positioning of sensors, the maxima were found at slightly different values of $U_{\mathrm{b}}$. These values are shown as dots in fig. 5 . The continuous lines give the analogous values as obtained by numerical simulations. The degree of agreement gives an indication of the reliability for individual sensors of the conversion factors and $\Delta E / E$ resolutions shown in table 1 .

\section{Analog and digital electronics}

A functional block diagram of the HARP electron: is presented in fig. 6. Since the twr hyperbolic analyzer heads have identical electronics, only one of them (HEAD-0) is shown in some detail.

The V-CAP generator controls the poiential of the end-cap electrodes and of the cover plate (CP). This potential can be changed within the limits of $\pm 20 \mathrm{~V}$ relative to the spacecraft. During most of the mission a zero value was maintained.

The potential of the body electrode of the analyzer is set by the HV-STEP bipolar voltage generator. The maximum number of potential steps is 75 between the limiting values of 0.6 and $1250 \mathrm{~V}$ (negative for electrons, positive for ions). The minimum energy increment of the analyzer is thus about $11 \%$. Requirements for the design included a $5 \%$ accuracy of the potentials throughout the allowed range, a $T=2 \mathrm{~ms}$ maximum time for a changeover from one potential to the next one, and a low power consumption.

The charged particles leave the analyzer with their original energy. A bipolar high-voltage generator (HVACC) accelerates the particles onto the MCP detector. The resulting cascade transfers a charge of 10 to $30 \mathrm{pC}$ onto one of the four anodes. The high voltage for the MCP (2200 to $3200 \mathrm{~V})$ is provided by the HV-MCP

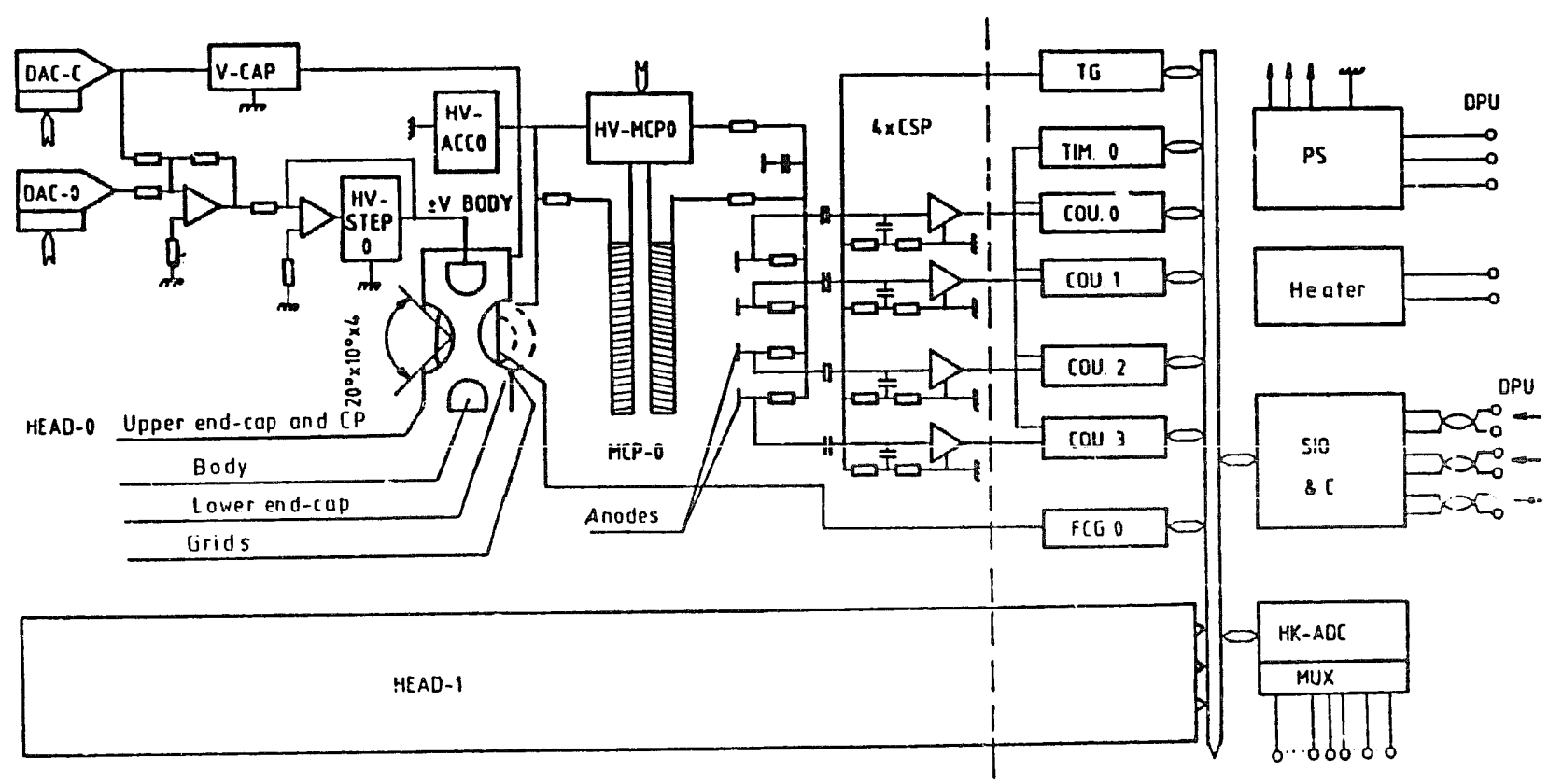

Fig. 6. Functional block diagram of the HARP electronics. For more details see section 4. 
unit. The MCP voltage had to be somewhat increased during the mission in order to compensate for the MCP degradation.

The anode signals are amplified by charge-sensitive preamplifiers (CSP). A discrimination level of $3 \mathrm{pC}$ was accepted after a study of the amplitude distribution and noise characteristics of the anode signals. The amplified signals are accumulated by counters (COU) during a time incerval preset by the timer (TIM), which can be programmed in the wide range of $10 \mathrm{~ms}$ to $1 \mathrm{~s}$, depending on the expected electron and ion fluxes. At the end of the integration time an interrupt is sent to the digital processing unit (DPU) and the contents of the eight counters are transmitted to the DPU through a serial data link. A new control potential (V-BODY) is set, the counters are cleared and the above procedure is restarted. At the end of a nicasurement sweep, housekeeping data are also transmi tted to the DPU.

The housekeeping data include 24 analog and 2 digital (status) bytes. The analog signals are selected and digitized (with 8-bit resolution) by the HK-ADC unit. These data include all high-voltage and supplyvoltage values, MCP and filament currents, current and voltage of the primary power line, and four characteristic temperatures.

The three instruments of the ESTER complex (LET, SLED and HARP) are served by the same DPU, which is located at a different part of the spacecraft. This unit is described in a separate paper [2].

The digital part of the HARP electronics has the task to:

- receive and decode the commands arriving from DPU,

- transmit the commands to subunits of HARP,

- transmit measurement and housekeeping data to DPU.

The communication with DPU i.: realized through a $153.6 \mathrm{kbit} / \mathrm{s}$ asynchronous serial data link (UART), galvanically separated by optocouplers. The internal data and address bus of the HARP is jointly controlled by UART and an internal synchronous sequential network (SIO\&C). A hardware optimization makes sure that the information exchang, between DPU and HARP requires as little DPU time as possible.

The power supply (PS) of HARP providing five different voltages is protected against short-circuits and excessive currents. If any of the voltages is below preset limits, a spare unit automaticalily takes over. A switchover can also be initiated by telecommand.

Instruments on-board the Phobos probes were designed for an active lifetime of at least $\mathbf{4 6 0}$ days. Extreme temperatures $\left(-50\right.$ to $+50^{\circ} \mathrm{C}$ ) and an estimated radiation dose of about $7 \mathrm{krad}$ were expected, with possible detrimental effects on the parameters of some sensitive components. In order to check proper functioning, an automatic self-test of HARP was planned for a complete measurement cycle each day. During the test an internal test generator (TG) of HARP sent a charge of $10 \mathrm{pC}$ onto the input point of each preamplifier, and then the whole measuring chain was tested. For testing the MCP, a filament is heated by the current generator FCG and serves as an electron source. The program changed the MCP voltage HV-MCP in 7 steps, thus providing the MCP characteristics.

For an enhanced reliability, radiation-sensitive components were shielded and placed near the centre of panels. Excessive cold was avoided by heating. At temperatures above $+50^{\circ} \mathrm{C}$, the program switched off HARP. Several circuits were duplicated.

\section{HARP software}

The HARP program is running under the RTS-800 multitask real-time supervisor of DPU. It has four major tasks:

- telecommand acknowledge and execution,

- in-flight calibration,

- automatic self-test,

- measurement control.

The measurements are defined by parameter tables called descriptors. There are six predefined descriptors burned into a PROM, containing parameter values intended for routine measurements during the interplanetary cruise phase and in the Mars environment. These predefined descriptors are easily available by telecommands, and any of them can be modified by additional commands. A modified descriptor can be stored in a protected bxitery-powered RAM area, and it is prestived even in the case of a switch-off. It is automatically used for the measurements after switch-on. If no descriptor is stored in that area, then the first of the predefined descriptors is used.

A descriptor consists of 12 bytes containing the following inforruation:

- type of measired particles (elecirons, ions, or both),

- lowest energy to be measured, energy increments (considerable versatility is allowed, with up to three energy regions sampled at different logarithmic energy steps,

- integration time,

- MCP voltage.

Under DPU control, the measured HARP data are organized into data frames. The smallest HARP frame contains counts obtained for 8 directions and 25 energy steps as well as housekeeping and status data. Such a data set occupies two telemetry blocks ( 240 bytes). Each count occupies 1 byte after data compression from 16 bits. The largest data frame contains 75 energy steps and can be transmitted in 6 telemetry blocks.

In the low bit-rate telemetry mode, two telemetry blocks (one HARP data frame) are transmittsd every 20 minutes. In the high bit-rate mode, usuallv four teleme- 
try blocks are transmitted once a minute. HARP also has a mass-memory for storing results for later transmission. A more detailed description of the HARP software is given in ref. [3].

\section{In-flight performance}

The HARP devices on both Phobos probes were switched on 12 days after the launch (launch dates: 7 July 1988 for Phobos 1, 12 July 1988 for Phobos 2). Both devices were operating during most of the active lifetime of the two Phobos probes (until 29 August 1988 for Phobos 1 and 26 March 1989 for Phobos 2). During the period when both probes were still active, electron flux variations detected by the two HARP devices matched reasonably well (a periect match is not expected because of the different spatial positions of the two probes). During the elliptical and circular orbits of Phobos 2 around Mars - particularly during the high bit-rate telemetry sessions for close encounters - important scientific data were gathered.

Fig. 7 illustrates the performance of the instrument just before and during the first encounter with Mars of the Phobos 2 spacecraft on 1 February 1989. Electron count rates as a function of energy (horizontal axis) are presented for a sequence of times. Data obtained for the four inner viewing sectors of the sensors are separately shown. Evidence for electron acceleration is clearly seen at and after the bow shock (18:25 UT), but some activity is also apparent in the foreshock region preceding the bow shock. These measurements agree fairly well with the results of the other instrument teams.
The energy spectra at low energies are influenced by the spacecraft potential and also by the geometry of the field in the environment of the instrument. The Debye length or shielding length in the interplanetary space is comparable to the dimensions of the whole spacecraft, and even at closest approach to Mars it is comparable to the distance to other instruments and structural elements of spacecraft. Thus, electrostatic fields may influence the trajectories of approaching low-energy particles in an unpredictable way. Although the potential of the HARP cap and covering plate can in principle be changed by telecommand relative to the spacecraft, at the unexpectedly high pericenter altitudes this would not have eliminated the distortion of the trajectories. In any case, particles above several $\mathrm{eV}$ are not likely to be significantly deflected by such fields.

\section{Conclusions}

The HARP hyperbolic electrostatic analyzers onboard the Phobos 1 and Phobos 2 spacecraft gathered interesting scientific information throughout the active period of the Phobos mission. It was thereby demonstrated that this type of instrument originally designed for terrestrial ionospheric research can be adapted for use in interplanetary space and around Mars.

\section{Acknowledgements}

The authors wish to express their thanks to all colleagues in Hungary, the USA and the USSR who helped

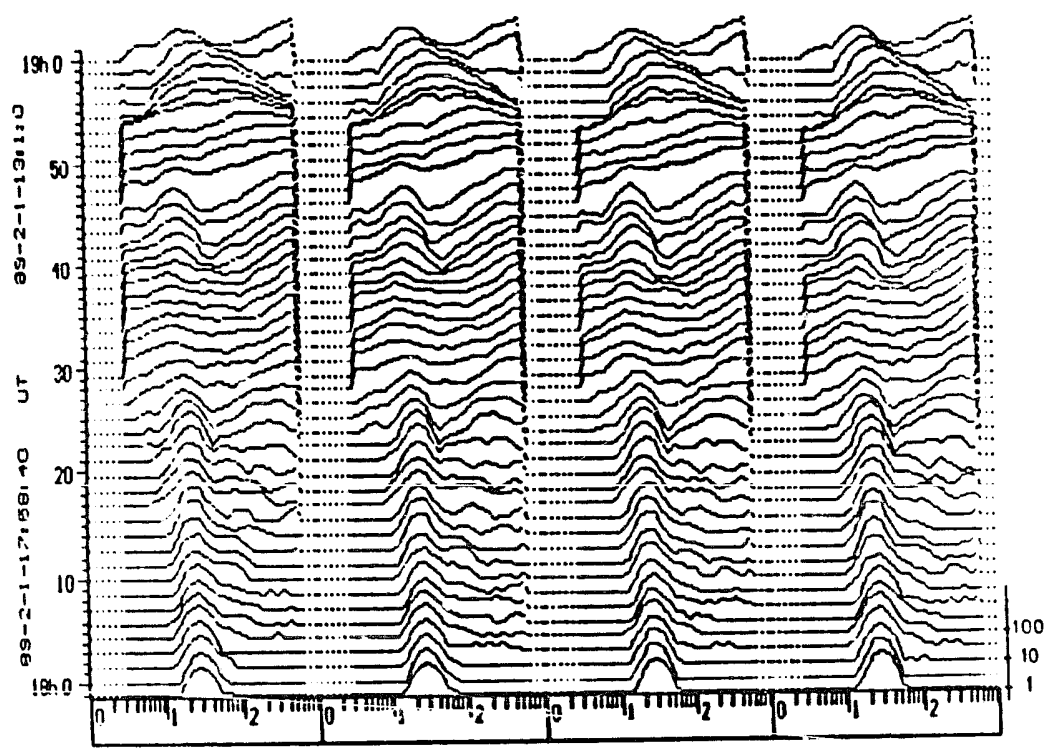

Fig. 7. Electron count rates detected by the four inner viewing sectors of the two sensors on Phobos 2 during the first encounter with Mars on 1 February 1989. The spacecraft crossed the bow shock at 18:25 UT. Closest approach to the surface of Mars (860 km) was at $18: 39$ UT. On the horizontal axis the logarithm of the electron energy (in $\mathrm{eV}$ ) is given. 
in the construction, calibration and data gathering of the HARP device. In particular, we would like to thank Drs. W. Chisholm, T.W. Shyn and I. Szentpétery for help in the calibration. NASA and the Hungarian Intercosmos Council are thanked for financial support given in the framework of an agreement on scientific collaboration.

\section{References}

[1] T.W. Shyn, W.E. Sharp and P.B. Hays, Rev. Sci. Instr. 47 (1976) 1005.

[2] V.V. Afonin et al., this issue, Nucl. Instr. and Meth. A290 (1990) 223.

[3] G. Endröczy, P. Király, L. Szabó, I. Sizemerey, S. Szendrö, I.T. Szücs, L. Várhalmi and J. Zsidó, Magyar Elektronika $5 / 9$ (1988) 17 , in Hungarian. 